\title{
Lunar Soil Strength Estimation Based on Chang'E-3 Images
}

\author{
Yang Gao ${ }^{1, *}$, Conrad Spiteri ${ }^{1}$, Chun-Lai Li ${ }^{2}$, Yong-Chun Zheng ${ }^{2}$
}

\begin{abstract}
Chang'E-3 (CE-3) was the third mission by China to explore the Moon which had landed two spacecraft, the CE-3 lander and Yutu rover on the lunar surface in late 2013. The paper presents analytical results of highresolution terrain data taken by CE-3's onboard cameras. The image data processing aims to extract sinkage profiles of the wheel tracks during the rover traverse. Further analysis leads to derivation or estimation of lunar soil physical properties (in terms of strength and stiffness) based on the wheel sinkage, despite the fact Yutu does not possess in-situ soil measurement instruments. Our findings indicate that the lunar soil at the CE-3 landing site has similar stiffness to what is measured at the Luna 17 landing site but has much less strength compared to the Apollo 15 landing site.
\end{abstract}

Keywords: Chang'E-3; Lunar rover; Photoclinometry; Soil stiffness; Terramechanics

\section{Introduction}

Following two lunar obiter missions Chang'E-1 and 2 commissioned in 2007 and 2010 respectively, the Chinese National Space Administration (CNSA) launched and landed the Chang'E-3 (CE-3) successfully on the northwestern Mare Imbrium of the Moon on the 14th December 2013 (Gao et al., 2014). CE-3 became the first soft landing on the Moon for China as well as for the

\footnotetext{
*Corresponding author

Email addresses: yang@surrey.ac.uk (Yang Gao), c.spiteri@surrey.ac.uk (Conrad Spiteri), lcl@bao.ac.cn (Chun-Lai Li), zyc@bao.ac.cn (Yong-Chun Zheng)

${ }^{1}$ STAR Lab, Surrey Space Centre, University of Surrey, Guildford, UK

${ }^{2}$ Key Laboratory of Lunar and Deep Space Exploration, National Astronomical Observatories, Chinese Academy of Sciences, Beijing, China
} 
world since the Apollo and Luna era. Figure 1a shows its landing location with respect to previous Moon landings by 1976. The CE-3 landing site is located on the east edge of a $430 \mathrm{~m}$ crater in the Sinus Iridum, adjacent to rather flat terrain with land forms such as craters, domes, strata and rocks that are good targets for scientific exploration (Chun-Lai Li et al., 2014).
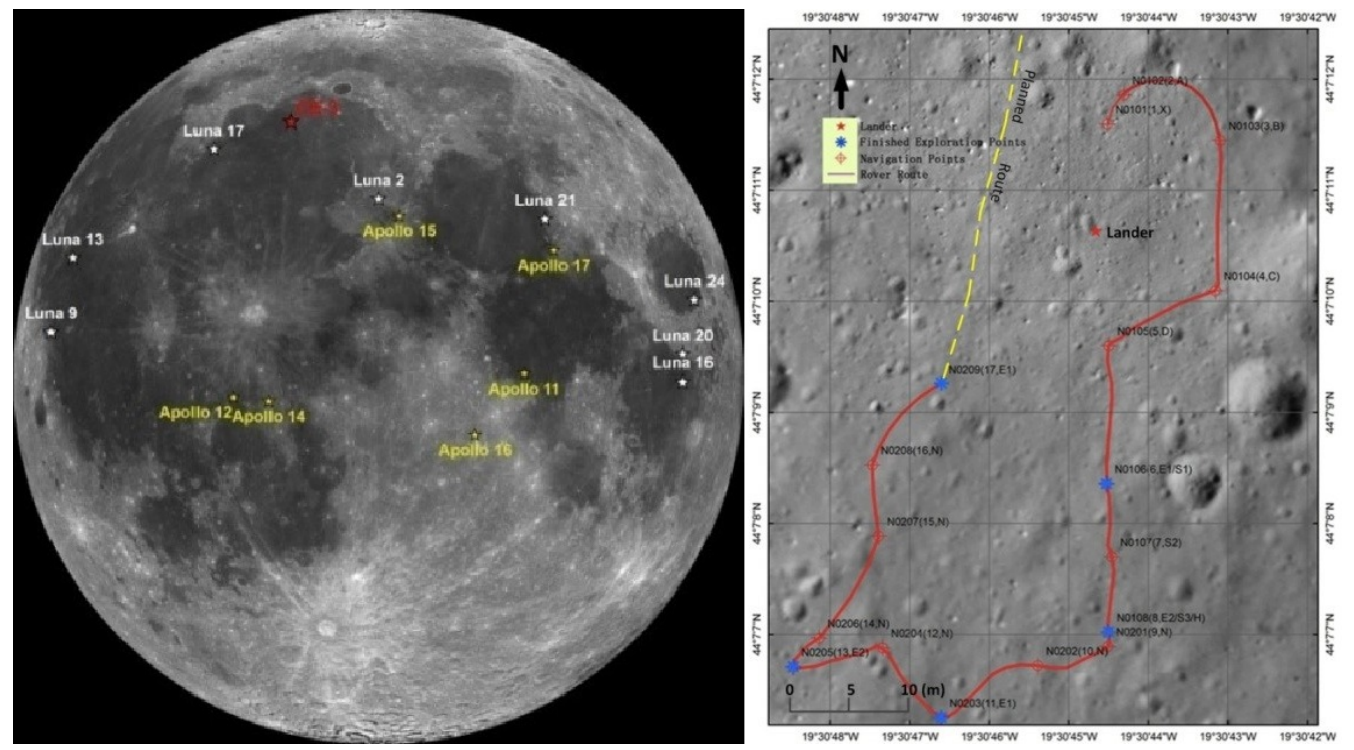

Figure 1: CE-3 soft landing and rover mission: (a) landing site w.r.t. the Luna and Apollo landings; (b) Yutu rover travel path.

Latest findings by Chinese lunar geologists also suggest that this region has experienced complex geological processes since the Imbrian and is compositionally distinct from the Apollo and Luna landing sites (Xiao L et al., 2015). It is therefore scientifically interesting to gain knowledge about the soil properties of this new landing area on the Moon, and to obtain data comparison between the CE, Luna and Apollo landing sites.

Lunar soil is known to be the fine fraction of the regolith found on the surface of the Moon. Due to difference in formation, the soil composition of the Moon differs significantly from that of Earth (Mitchel et al., 1972). Lunar soil's physical properties are primarily influenced by mechanical disintegration of basaltic and anorthositic rocks, caused by continuous meteorite impact and bombardment by interstellar charged atomic particles over billions of years (Chunlai Li et al., 2015). Understanding the Lunar soil properties has been of interest to both lunar geologists and engineers and can help with 
landing site selection, design and sizing of the rover, drill and sampler, etc.

Unlike several Luna and Apollo landing spacecraft, the CE-3 lander and rover called Yutu (Jade rabbit) were not equipped with any dedicated soil probe. Notable terrain data of the CE-3 landing site has been obtained by the lander's onboard terrain camera as well as a pair of panoramic cameras (PanCam) on-board Yutu. These cameras have captured high resolution (up to sub-centimeter) images of the lunar terrain during the rover traverse. These terrain images had been used for interpreting rover path in conjunction with data of the orbital imagery and rover wheel odometry. Figure $1 \mathrm{~b}$ (Chunlai Li et al., 2015) illustrates that the Yutu traveled from and around the lander covering approximately $40 \mathrm{~m}$ by $50 \mathrm{~m}$ area before it encountered mechanical fault. The rest of this paper presents an alternative application of the PanCam images for indirect measurement of the rover wheel sinkage leading to the derivation of planetary soil properties.

\section{Soil Analysis Methodologies \& Results}

Two scientific methods built on planetary robotics and terramechanics have been proposed and presented for estimating planetary soil stiffness: 1) the first, traditional method uses penetrometer data and Bernstein-Goriatchkin (BG) model to establish the pressure-sinkage relationship hence quantify the soil strength level. Its feasibility is demonstrated using Luna 17 and Apollo 15 penetrometer data; 2) the second, modern method investigates the rover wheel-soil interaction captured by images containing wheel tracks, and applies photoclinometry to extract wheel sinkage. The small wheel model can then be used to work out the stiffness ratio for the soil. The modern method has been tested on rover track images of Luna 17 and Apollo 15 so that its results are validated for consistency with the traditional method. Soil strength at the latest CE-3 landing site is also produced using the modern method and compared with the results found for the Luna 17 and Apollo 15 landing sites. Our findings indicate that Yutu experienced sinkage in the range of 0.5 to $1 \mathrm{~cm}$ given a total vehicle mass of $135 \mathrm{~kg}$.

\subsection{Traditional method}

The Luna 17 and Apollo 15 mission in the 1960-70's used an instrument called a penetrometer to measure soil strength and stiffness at different depths and locations around their respective landing sites (refer to Fig. 1). These are key measures in soil mechanics that determine whether 
or not soil will be stable or how much it will deform. The penetrometer data can produce plots of pressure (or stress) $p$ versus sinkage $z$ that the device experienced. In classical terramechanics, a mathematical formula like the Bernstein-Goriatchkin (BG) model (Bekker., 1956) is often used to estimate and predict the $p-z$ behaviors of static penetration within soil, which can be described as

$$
p=k z^{n}
$$

where $k$ is the stiffness modulus and $n$ is the sinkage exponent. In this study, we propose use of the BG model to correlate the $p-z$ plot of the penetrometer data which then produces the $k$ and $n$ values to quantify the soil strength. To demonstrate feasibility of the method, representative $p$ - $z$ data taken by the penetrometers has been re-gathered from (Heiken et al., 1991) for Luna 17 and from (Apollo 15 mission , 1971) for Apollo 15, and correlated against the BG model for the two landing sites.

\begin{tabular}{lllll}
\hline Landing Site & $\begin{array}{l}\text { No. of sampling } \\
\text { locations }\end{array}$ & $\begin{array}{l}z \\
(\mathrm{~cm})\end{array}$ & Median n & $\begin{array}{l}\text { Median } \\
k\left(\mathrm{~N} / \mathrm{cm}^{2+n}\right)\end{array}$ \\
\hline Luna 17 & 4 & $0-8.0$ & 0.54 & 0.8 \\
Apollo 15 & 6 & $0-15.7$ & 0.50 & 5.00 \\
\hline
\end{tabular}

Table 1: Traditional soil stiffness analysis based on penetrometer data and BG model

Results are summarized in Table 1. Penetrometer data from four and six sampling locations of the Luna 17 and Apollo 15 respectively have been used. Among these data, the penetrometer reached maximum depth of 8.0 and $15.7 \mathrm{~cm}$ for Luna 17 and Apollo 15 respectively. The sinkage exponent $\mathrm{n}$ derived has a median value of 0.54 and 0.50 respectively for the two landing sites. The two exponent values have less than $1 \%$ difference meaning that the two fitted $p-z$ plots mostly coincide given $z$ is a small value. Therefore, the $k$ value derived by the method can be considered a monolithic indicator for soil stiffness, the higher the value, the stiffer the soil. As shown in Table 1 , the stiffness modulus $k$ for the Apollo 15 is an order of magnitude higher than that of Luna 17, showing higher soil strength at the Apollo 15 landing site. 


\subsection{Modern method}

The Yutu rover is not equipped with a dedicated soil measurement instrument like the penetrometer, while its wheels were constantly in contact with soil and left tracks on lunar surface. Through these tracks, the wheel sinkage can be retrieved using photoclinometry or shape from shading (Gao et al., 2013; Spiteri et al., 2015). Similar approaches have been investigated by (Arvidson et al. , 2003, 2004; Herkenhoff et al. , 2008; Richter et al. , 2006), however these methods mostly require the use of stereo images. Such methods are typically associated with high computational costs and hence, are unable to operate in Real-Time (Gao et al., 2013). Some Shape from Shading algorithms are able to closely replicate results obtained through stereo methods and can be designed to consume less computational resources (Gao et al., 2013; Spiteri et al., 2015).

Developed by Horn in the early 70's (Horn , 1970) and inspired by biological systems (Ramachandran , 1988), photoclinometry or Shape from Shading (SfS) is a method that attempts to extract the three dimensional shape of an object or scene from a 2D grey scale image using the pixel intensities and light direction as an indication of the surface gradient, surface normal or depth. It is fundamentally based on the Lambertian reflectance model and Lambert's cosine law (Horn , 1970).

In reality, SfS reconstruction needs to make assumptions on the object's geometry, albedo, reflectance properties and image formation process. Although such approaches were demonstrated to generate reasonable 3D models, they all impose limitations on their applicability to real-world images (Cremers , 2012). However, such statement bears only natural terrestrial images into account, where a diversity of object types, albedos and reflectance properties may be encountered at varying degrees within an image.

Planetary natural images such as the ones taken on the Moon and Mars have a restricted set of object properties, acute albedo variations and negligible reflectance diversities (Wilhelms , 1970; Taguchi et al., 2014; Biswas

et al., 2007). These constraints make it an ideal scenario for reconstructing the scene's DTM using SfS techniques. In fact, SfS methods have been extensively used with single high resolution satellite imagery to optimize the interpolation techniques used in creating planetary DTMs including the Mars High Resolution Imaging Science Experiment (HiRISE) (Rajabi et al., 2004).

Algorithms for various SfS methods have been evaluated and benchmarked against each other in literature (Zhang et al., 1999), however these mostly 
use generic Earth based images or satellite planetary images. In order to determine the most competent algorithm for planetary rovers, these algorithms have been assessed using planetary images or datasets that resemble a planetary environment, such as Mars and the Moon, on Earth through the eyes of a rover. As a result, it has been determined that a linear approximation method as presented by Tsai and Shah (Tsai \& Shah , 1994), gave the most accurate results at the fastest execution speed. This is in line with Zhang et al. results (Zhang et al., 1999). The algorithm employs discrete approximation of the gradient first, then a linear approximation of the reflectance map is used to extract the depth using Jacobi iterative schemes. The linear approximation method used also assumes uniform albedo since it is envisaged that the regolith being investigate is mostly of the same type with minimal albedo deviation.

A PanCam image of Yutu can be seen in Figure 2a which has a resolution of 2352 by 1728 pixels and a depth of 10 bits encapsulated in a Planetary Data System (PDS) format. Metadata considered by our method includes the rover's pose with relation to the lander, orientation of the PanCam cameras, image parameters, wheel odometry and sun elevation and azimuth. The PSD file also indicates fixed camera parameters such as the $50 \mathrm{~mm}$ focal length and $7.4 \mu \mathrm{m}$ pixel size.

The image sections that contain rover tracks can be analyzed to extract the track depth or wheel sinkage $z$, at various locations along the rover path. An example of the image section is shown in Figure $2 b$ (which has been scaled up for readability purpose) whose position is highlighted by the red box on the original PanCam image in Figure 2a. A linear approximation photoclinometry algorithm processes the section to model the terrain topology that outputs a Digital Terrain Model (DTM) in 3-dimensional pixel scale as seen in Figure 2c where the $z$-axis is illustrated using color codes along the side. Viewing the DTM in the heading direction of the rover (i.e. the y-axis in Figure $2 \mathrm{~b} \& 2 \mathrm{c}$ ), distance between the lowest point of the DTM for the tracks and the surface elevation represents wheel sinkage $z$ for any given location along the rover path. Using the pinhole camera model, the wheel sinkage $z$ is quantified using:

$$
z=\frac{p l_{z} d}{f}
$$

where $p l_{z}$ is the total pixel length of the sinkage depicted in Figure 2c, $d$ is the distance value between the camera and the sinkage location along the 
rover path given by the wheel odometry, and $f$ is the focal length of the camera.

In consequence of the wheel design, each individual wheel of the Yutu rover can leave up to three track marks in the soil. Depending on how well the wheel is in contact with the ground, the three track marks can show different level of sinkage. Yutu, similarly to most planetary rovers, uses a rocker-bogie suspension that seeks to maintain wheel contact and equalize rover weight carried by each wheel. This set of passive linkages with no elastic elements ensures all wheels maintain contact with the ground. This low mass solution ensures near equal weight distribution over all wheels on uneven terrain, dramatically improving mobility capability. (Huber S., 2009; Senatore et al. , 2011)

Figure $2 \mathrm{~d}$ shows the sinkage profiles for two visible track marks based on data processing from Figure $2 \mathrm{~b}$ and 2c. As a general rule of thumb, the highest-valued sinkage profile is considered as the overall wheel sinkage because it indicates the best contact with the soil and therefore abate the effect of a non-constant value of normal stress across the wheel-soil contact area. This also abates the issue that the wheels are not cylindrical in shape and are a close resemblance to a prolate spheroid.

Additionally, multiple passes of wheels on the same rut by consecutive wheels is ignored since the aim of the paper is to compare soil properties, in terms of stiffness and strength, to that of other lunar sites, from images taken by different rovers and methods. The ruts left by wheels from different missions have also experienced a minimum of two wheel passes and as per the study by Holm the increase in rut depth after the second wheel pass is minimal to negligible (Holm , 1969). Hence for Figure 2d, the sinkage profile induced by the middle track mark of the wheel is used. The track marks left by wheels on the other side of the rover are processed in a similar manner. As a result, the higher-valued sinkage between the right and left side of the rover wheels is considered the overall wheel sinkage $z$.

A modern $p$ - $z$ model in terramechanics works on sinkage caused by circular wheels of a small vehicle like Yutu. The pressure $p$ encountered by the rover wheel is calculated by $M g / A$ (Meirion et al., 2011), where $M$ is the mass of the vehicle divided by the total number of wheels assuming even distribution of pressure among the wheels, $g$ is the Moon gravity $1.633 \mathrm{~m} / \mathrm{s}^{2}$, and $A$ is the contact area of the wheel with the soil and calculated by: 


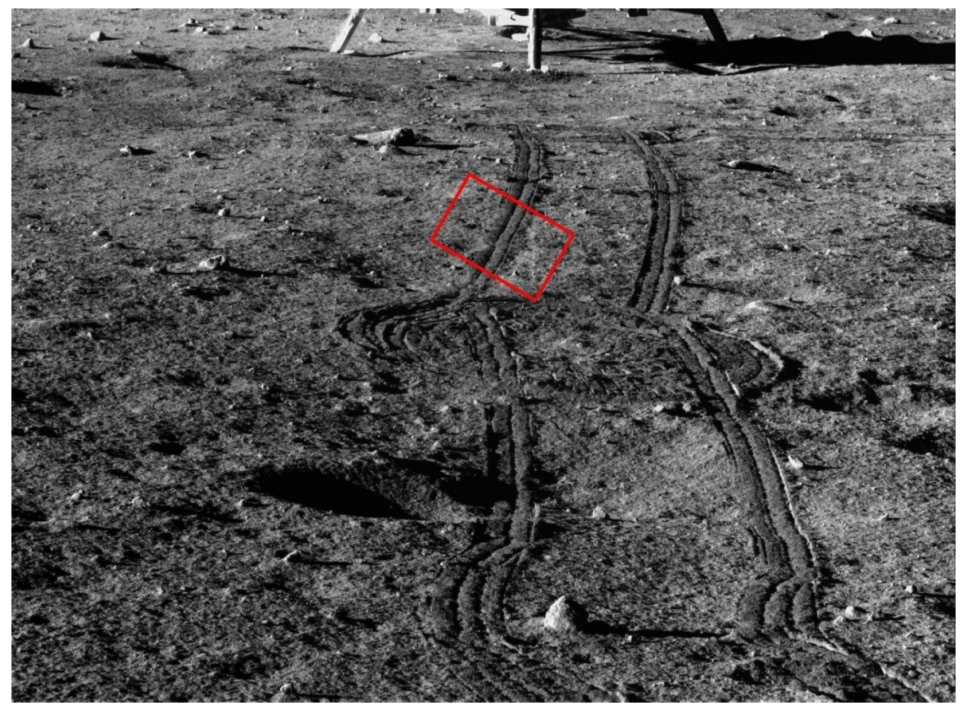

(a)

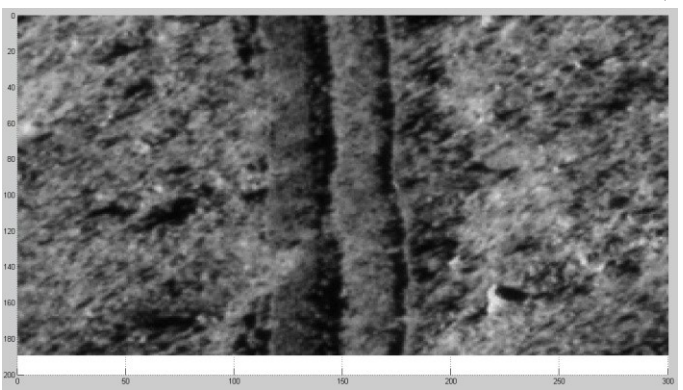

(b)

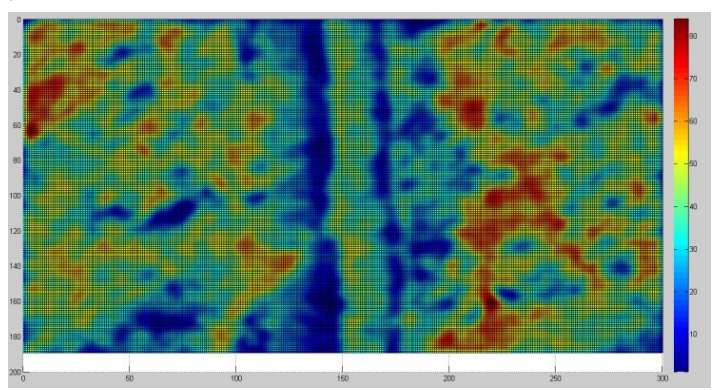

(c)

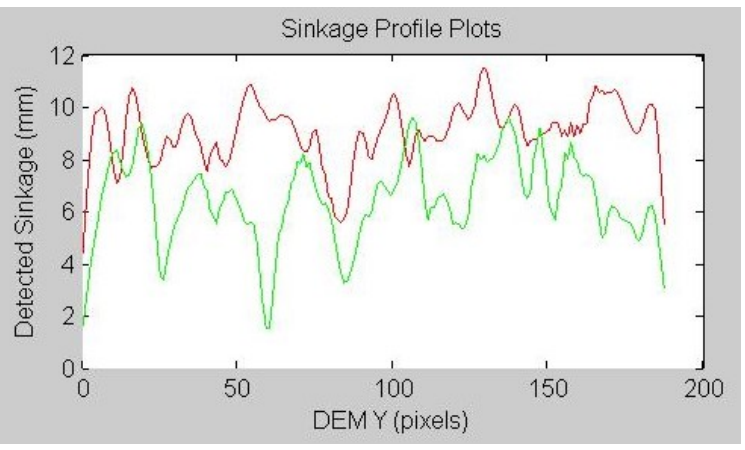

(d)

Figure 2: Rover wheel sinkage extraction: (2a) original Yutu PanCam image; (2b) image section containing rover track marks in 2-dimentional pixel scale, corresponding to the red box region in 2a; (2c) DTM of the image section in 3-dimentional pixel scale where the z-axis values are illustrated in color codes; (2d) dynamic sinkage profiles extracted from the image section for the middle and right track marks. 


$$
W \operatorname{Ros}^{-1}\left(\frac{R-z}{R}\right)
$$

where $W$ is the wheel width, $R$ is the wheel radius and $z$ is the calculated sinkage. The small wheel model (Meirion et al., 2011) has modified the traditional BG model by taking into account the wheel effect and is described as

$$
p=k D^{m} z^{n}
$$

where $D$ is the wheel diameter and $\mathrm{m}$ is the diameter exponent. The $n$ and $m$ values in (Meirion et al., 2011) were derived for terrestrial dry sand, however, (Mitchel et al., 1972), in their study on Lunar soil samples, noted that although the lunar soil properties is compositionally different to that of terrestrial dry sand, the mechanical properties are remarkably similar. Therefore, for dry sand which is applicable to existing lunar landing sites, the model suggests $n=0.8$ and $m=0.39$. Given $n$ and $m$ are fixed in this model, the $p-z$ relation is driven by both $k$, the stiffness modulus and $D$, the size of the wheel. Since the wheel sinkage $z$ can be calculated using the data processing method described above, hence $p$ based on the formula, the $k$ indicating the soil stiffness can then be calculated.

To demonstrate the method, we have analyzed image data containing rover tracks for CE-3 as well as the Luna 17 and Apollo 15 missions that were considered in Section 2.1. To do so, we can potentially cross reference and validate the modern method by comparing results drawn by the traditional method. Here track images of the 8-wheeled Lunokhod-1 rover in Luna 17, the 4-wheeled Lunar Roving Vehicle (LRV) in Apollo 15 and the 6-wheeled Yutu rover in CE-3 have been analyzed. Results are summarized in Table 2. The wheel sinakge profiles extracted for the three vehicles where each gives a range shown in the $z$ column. The physical properties of the three vehicles in terms of $M, D$ and $A$ are given in their respective columns allowing $p$ to be further computed. Given sinkage is calculated as a dynamic profile using this method, the corresponding sinkage modulus $k$ is thus also dynamically changing. For comparison purposes, the median value of $k$ is also considered similar to using the traditional method. The corresponding $k D^{0.39}$ values are given in the last column of the Table 2 which indicates the overall $p-z$ relations.

Mathematical, analytical results using the traditional method based on the BG model in Eq. 1 or the modern method based on the small wheel 
model in Eq. 4 are further illustrated in Figure 3, where the resulted model plots estimating the $p-z$ relationship are presented. It is evident that both methods have led to numerically comparable and similar models for Luna 17 and Apollo 15 to estimate soil stiffness. Both methods also conclude that the soil strength at the Apollo 15 landing site is much greater than Luna 17. The modern method further reveals that CE-3 has a same level of soil stiffness as the Luna 17 hence less strength compared to the Apollo 15 landing site.

\begin{tabular}{|l|l|l|l|l|l|l|l|l|}
\hline $\begin{array}{l}\text { Landing } \\
\text { site }\end{array}$ & Vehicle & $\begin{array}{l}\text { Track } \\
\text { distance } \\
\text { covered }(\mathrm{m})\end{array}$ & $\begin{array}{l}\text { Vehicle } \\
\text { mass per } \\
\text { wheel } M(\mathrm{Kg})\end{array}$ & $\begin{array}{l}\text { Wheel } \\
\text { diameter, } \\
D(\mathrm{~cm})\end{array}$ & $\begin{array}{l}\text { Wheel-soil } \\
\text { contact area } \\
A\left(\mathrm{~cm}^{2}\right)\end{array}$ & $\begin{array}{l}p=M g / A \\
\left(\mathrm{~N} / \mathrm{cm}^{2}\right)\end{array}$ & $\begin{array}{l}z \\
(\mathrm{~cm})\end{array}$ & $\begin{array}{l}\text { Median } \\
k D^{0.39} \\
\left(\mathrm{~N} / \mathrm{cm}^{2.8}\right)\end{array}$ \\
\hline \hline Luna 17 & Lunokhod-1 & $2-3$ & 94.50 & 51 & $148.26-229.56$ & $0.67-1.04$ & $1.07-2.54$ & 0.5 \\
\hline Apollo 15 & LRV & $4-5$ & 177 & 82 & $126.78-295.01$ & $0.98-2.28$ & $0.37-1.99$ & 2.45 \\
\hline CE-3 & Yutu & $6-7$ & 22.50 & 30 & $61.67-88.68$ & $0.41-0.60$ & $0.56-1.15$ & 0.55 \\
\hline
\end{tabular}

Table 2: Traditional soil stiffness analysis based on penetrometer data and BG model

It is worth noting that the main difference between the plots produced by the two methods is driven by the difference of the sinkage exponent $n$. The traditional method derives the $n$ values based on penetrometer measurements which are 0.50 .6 for both the Luna 17 and Apollo 15 landing sites, while the modern method uses a fixed value 0.8 for dry sand out of a rule of thumb. Future work could investigate further how to determine $n$ in the modern method more intuitively.

\subsection{Remarks}

The two analytical methods offer consistent results allowing both to be used in planetary missions depending on available payload resources. The traditional method works directly on penetrometer payload data allowing soil stiffness to be quantified for different sampling locations. The modern method can produce dynamic analysis of soil stiffness over the planetary surface. It takes advantage of typical imagery data containing vehicle tracks without dedicated in-situ soil sensing. CNSA is currently developing followup missions of CE-3 where improved understanding of lunar soil mechanics is important. For examples, the CE-4 plans to land and rove on the far side of the Moon before 2020; the CE- 5 will be a lunar sample return mission planned for launch in 2017. These future missions are envisaged to use (or benefit from) results of this study while developing relevant scientific experiments and engineering tasks such as sampling lunar soil. 


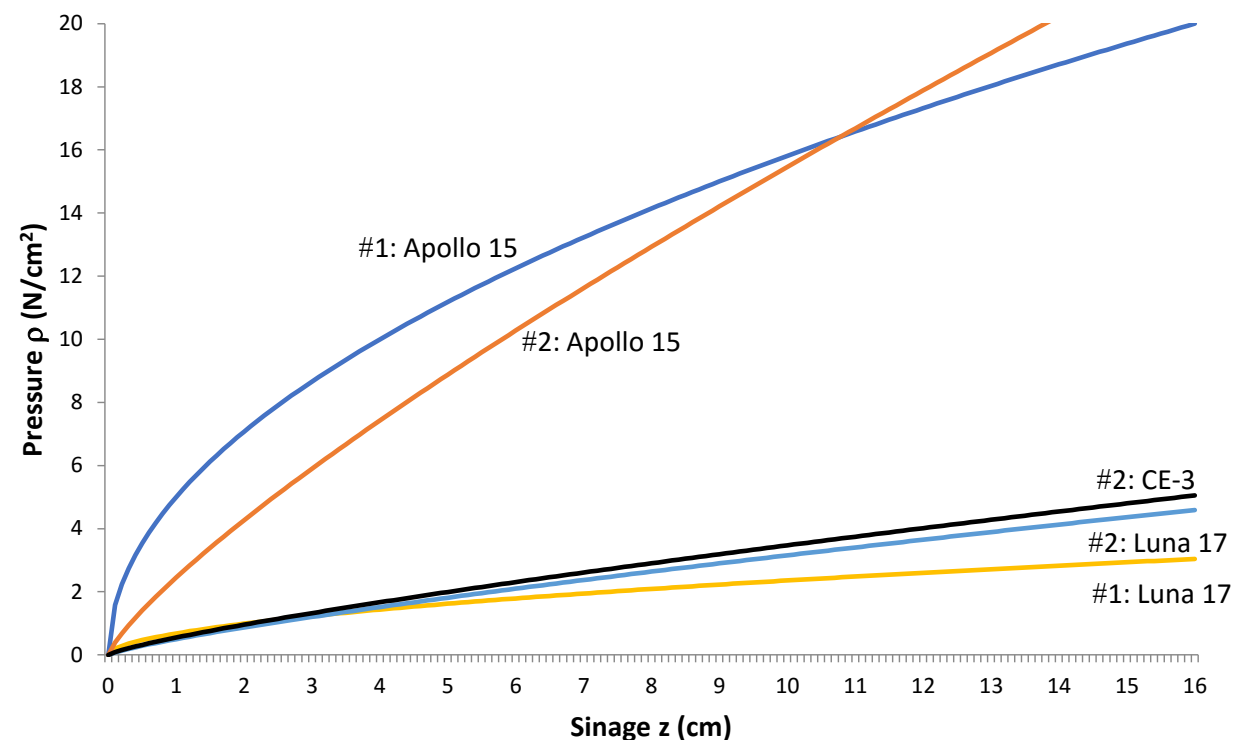

Figure 3: The $p-z$ model plots based on traditional method and modern method for Luna 17, Apollo 15 and CE-3, illustrating final numerical results in Tables 1 \& 2 . \#1 refers to the traditional method (2.1) \#2 refers to the modern method (2.2).

\section{Conclusions}

The paper presents two multidisciplinary, analytical approaches for quantifying soil strength using pressure-sinkage relation for planetary bodies. The two methods have been crossed referenced and verified with in-situ soil penetrometer data from Apollo 15 and Luna 17. The modern method in particular allows generation of the first results of CE-3 mission on lunar soil analysis. The main findings can provide reference to lunar soil mechanics for in-depth studies of the Moon and support future missions within the Chang'E program and others.

\section{Acknowledgements}

The project is funded by the UK Royal Academy of Engineering under the international research exchange major award. 


\section{References}

Gao, Yang \& Liu, Jinguo; China's robotics successes abound: American Association for the Advancement of Science, Volume: 345, No. 6196, pages: $523-523,2014$

Chun-Lai Li, Ling-Li Mu, Xiao-DuanZou, Jian-Jun Liu, XinRen, XingGuoZeng, Yi-Man Yang, Zhou-Bin Zhang, Yu-Xuan Liu ,Wei Zuo, Han $\mathrm{Li}$, Analysis of the geomorphology surrounding the Chang'E-3 landing site. Research in Astronomy and Astrophysics, Vol 14, Issue 12, ISSN 1674-4527, 2014.

Xiao L, Zhu P, Fang G, Xiao Z, Zou Y, Zhao J, Zhao N, Yuan Y, Qiao L, Zhang X, Zhang H, Wang J, Huang J, Huang Q, He Q, Zhou B, Ji Y, Zhang Q, Shen S, Li Y, Gao Y, A young multilayered terrane of the northern Mare Imbrium revealed by Chang'E-3 mission, Science, 347(6227):1226-9, 2015.

Chunlai Li, Jianjun Liu, Xin Ren, Wei Zuo, Xu Tan, Weibin Wen, Han Li , Lingli Mu, Yan Su, Hongbo Zhang, Jun Yan, Ziyuan Ouyang, The Chang'e 3 Mission Overview, Space Science Reviews, Volume 190, Issue 1, pp 85-101, July 2015.

Bekker, M.G., 1956. Theory of land locomotion-the mechanics of vehicle mobility - University of Michigan Press.

Heiken Grant, David Vaniman, and Bevan M. French, eds. Lunar sourcebook: A user's guide to the Moon (Chapters 4 and 7), CUP Archive, 1991.

Apollo 15 penetrometer data archive: http://pds-geosciences.wustl. edu/lunar/a151-1-1srp-2_3-soil-mechanics-v1/a15sm_0001/data/

Yang Gao, Conrad Spiteri, Minh-Tri Pham, and Said Al-Milli, A Survey on Recent Object Detection Techniques Useful for Monocular Vision-based Planetary Terrain Classification, Robotics and Autonomous Systems, Vol. 62, Issue 2, pp. 151-167, 2013.

Conrad Spiteri, Yang Gao, Said Al-Milli, and Aridane Sarrionandia de Len, Real-time Visual Sinkage Detection for Planetary Rovers, Robotics and Autonomous Systems, Vol: 72, pp. 307-317, October 2015. 
Gareth Meirion and Matthew Spenko A Modified Pressure-Sinkage Model for Small, Rigid Wheels on Deformable Terrains, Journal of Terramechanics, 48, 149-155, 2011.

C. Senatore, C. Sandu, Off-road tire modeling and the multi-pass effect for vehicle dynamics simulation, Journal of Terramechanics, Volume 48, Issue 4, August 2011, Pages 265-276, ISSN 0022-4898.

Steven A. Huber, Conjoined Lander-Rovers for Planetary Exploration

Horn, B. K.P., SHAPE FROM SHADING: A METHOD FOR OBTAINING THE SHAPE OF A SMOOTH OPAQUE OBJECT FROM ONE VIEW, Massachusetts Institute of Technology, 1970.

Ramachandran, V. S., Perceiving Shape From Shading, Scientific American, Issue 8 volume 256, August 1988.

Cremers, Daniel, Fast and Globally Optimal Single View Reconstruction of Curved Objects, Proceedings of the 2012 IEEE Conference on Computer Vision and Pattern Recognition (CVPR) 2012.

Wilhelms, Don E. Auteur, Summary of lunar stratigraphy - Telescopic observations - Contributions to Astrogeology, Geological Survey Professional Paper; 599-F, Washington DC 1970

Taguchi, M., Funabashi, G., Watanabe, S., Takahashi, Y., Fukunishi, H., Lunar albedo at hydrogen Lyman $\alpha$ by the NOZOMI/UVS, Journal of Earth, Planets and Space, volume 52, number 9, pages 645-647, 2014.

Biswas, Soma and Aggarwal, Gaurav and Chellappa, Rama, Robust Estimation of Albedo for Illumination-invariant Matching and Shape Recovery, IEEE Computer Society. pages 1-8, 2007

Rajabi, Mohammad A. and Rod Blais, J. A., Optimization of DTM Interpolation Using SFS with Single Satellite Imagery, The Journal of Supercomputing, volume 28, number 2, pages 193-213, 2004.

, Ruo Zhang and Ping-Sing Tsai and James Edwin Cryer and Mubarak Shah, Shape from Shading: A Survey, IEEE TRANSACTIONS ON PATTERN ANALYSIS AND MACHINE INTELLIGENCE, volume 21, number 8, pages 690-706, 1999. 
Tsai Ping-Sing and Mubarak Shah, Shape from shading using linear approximation, Image and Vision Computing, volume 12, number 8, pages 487 498, 1994.

I.C. Holm, Multi-pass behaviour of pneumatic tires, Journal of Terramechanics, Volume 6, Issue 3, 1969, Pages 47-71

R.E. Arvidson et al. (2003). Physical Properties and Localization Investigations Associated with the 2003 Mars Exploration Rovers. J. Geophys. Res. 108 E12, 8070, doi: 10.1029/2002JE002041.

K. E. Herkenhoff et al. (2008). Chapter 20: In Situ Observations of the Physical Properties of the Martian Surface. In: Jim Bell (ed.). The Martian Surface: Composition, Mineralogy, and Physical Properties. Cambridge University Press.

R.E. Arvidson et al. (2004). Localization and Physical Properties Experiments Conducted by Spirit at Gusev Crater. Science, Vol 305, Issue 5685, pp. 821-824.

L. Richter, A. Ellery, Y. Gao, S. Michaud, N. Schmitz, S. Wei (2006). A Predictive Wheel-Soil Interaction Model for Planetary Rovers Validated in Testbeds and Against MER Mars Rover Performance Data. Proceedings, 10th European Conference of the International Society for Terrain-Vehicle Systems (ISTVS), Budapest, Oct. 3-6.

Irom, F. and Swift, G.M. and Farmanesh, F.H. and Johnston, A.H., Singleevent upset in commercial silicon-on-insulator PowerPC microprocessors, SOI Conference, IEEE International 2002 pages 203-204

Mitchell J. K. and Houston W. N. and Scott R. F. and Costes N. C. and Carrier III, W. D. and Bromwell L. G., Mechanical properties of lunar soil: Density, porosity, cohesion and angle of internal friction, Lunar and Planetary Science Conference Proceedings, volume 3, 1972 